\title{
Prática educativa na área da saúde da mulher: relato de experiência
}

\author{
Educational practice in the women's health area: experience report \\ Práctica educativa en el área de salud de la mujer: relato de experiencia
}

Recebido: 13/08/2021 | Revisado: 22/08/2021 | Aceito: 29/08/2021 | Publicado: 31/08/2021

\author{
Matheus Almeida Ramalho \\ ORCID: https://orcid.org/0000-0002-5607-1313 \\ Universidade Federal de Jataí, Brasil \\ E-mail: matheusramalho@ discente.ufg.br \\ Joyce Francelino Silva \\ ORCID: https://orcid.org/0000-0003-1267-7361 \\ Universidade Federal de Jataí, Brasil \\ E-mail: joyce.francelino@discente.br \\ Jaqueline Ferreira de Miranda \\ ORCID: https://orcid.org/0000-0003-3945-9399 \\ Universidade Federal de Jataí, Brasil \\ E-mail: jaqueline.ferreira@discente.ufg.br \\ Lázara Bianca Oliveira Sousa \\ ORCID: https://orcid.org/0000-0003-4887-1289 \\ Universidade Federal de Jataí, Brasil \\ E-mail: lbianca@discente.ufg.br \\ Keila Pereira de Assis \\ ORCID: https://orcid.org/0000-0002-5064-9866 \\ Universidade Federal de Jataí, Brasil \\ E-mail: keilaassis@ discente.ufg.br \\ Hellen Cristina Sthal \\ ORCID: https://orcid.org/0000-0001-8139-992X \\ Universidade Federal de Jataí, Brasil \\ E-mail: hcsthal@ufj.edu.br \\ Giulena Rosa Leite \\ ORCID: https://orcid.org/0000-0002-9294-0985 \\ Universidade Federal de Jataí, Brasil \\ E-mail: giulenar@ufg.br
}

\begin{abstract}
Resumo
Introdução: Ações de promoção à saúde da mulher devem ser oferecidas de modo holístico, participativo, individual e coletivo, em todos os âmbitos. Considerando que a mulher deve ser orientada sobre autocuidado para que, de forma consciente e autônoma, gerencie seus cuidados de saúde. Objetivo: Relatar a experiência na realização de uma ação de educação em saúde, referente à saúde da mulher. Metodologia: Estudo descritivo, do tipo relato de experiência, realizado por acadêmicos de Enfermagem da UFJ, a respeito do evento OAB Mulher. Resultados e Discussão: O evento ocorreu em março de 2019, contando com palestras, uma feira de saúde e beleza, espaço jurídico e psicológico, espaço infantil, almoço e abertura para lazer no clube Thermas. No projeto houve a participação de dez discentes e duas docentes do curso de Enfermagem, foram desenvolvidas práticas educativas relativas ao planejamento familiar, sexo seguro, prevenção de ISTs, gravidez na adolescência, autocuidado e a importância de um estilo de vida saudável e debate sobre violência. Conclusão: $\mathrm{O}$ evento proporcionou aos discentes o contato com a realidade social das participantes, possibilitando a reflexão no que se refere à humanização na saúde da mulher e à compreensão das práticas pautadas no diálogo, no acolhimento e na construção coletiva.
\end{abstract}

Palavras-chave: Saúde da mulher; Prevenção de doenças; Educação em saúde.

\begin{abstract}
Introduction: Actions to promote women's health must be offered in a holistic, participatory, individual and collective way, in all areas. Considering that women should be guided on self-care so that, in a conscious and autonomous way, they manage their health care. Objective: To report the experience in carrying out a health education action, referring to women's health. Methodology: Descriptive study, the type of experience report, carried out by Nursing students at UFJ, regarding the Woman OAB event. Results and Discussion: The event took place in March 2019, with lectures, a health and beauty fair, legal and psychological space, children's space, lunch and leisure opening at the Thermas club. In the project there were the participation of ten students and two professors of the Nursing course, educational practices were developed regarding family planning, safe sex, STIs prevention, teenage pregnancy, self-care and the importance of a healthy lifestyle and debate about violence. Conclusion: The event provided the students the contact
\end{abstract}


with the social reality of the participants, enabling reflection with regard to humanization in women's health and the $u$ nderstanding of practices based on dialogue, welcoming and collective construction.

Keywords: Women's health; Prevention of diseases; Health education.

\begin{abstract}
Resumen
Introducción: Las acciones que promueven la salud de la mujer deben ofrecerse de manera integral, participativa, individual y colectiva, en todos los ámbitos. Debe de informarse a las mujeres sobre su autocuidado para que puedan gestionar, de manera consciente y autónoma, su atención sanitaria. Objetivo: Informar sobre la experiencia de educación sanitaria en la promoción de salud de la mujer. Metodología: Estudio descriptivo, de informe de experiencia, implementado por estudiantes de Enfermería de la UFJ, sobre el evento OAB Mujer. Resultados y Discusión: El evento tuvo lugar en marzo de 2019, contó con conferencias, una feria de salud y belleza, un espacio legal y psicológico, un espacio infantil, almuerzo y apertura del espacio del ocio en el club Thermas. El proyecto contó con la participación de diez estudiantes y dos profesoras del curso de Enfermería, se desarrollaron prácticas educativas en materia de planificación familiar, sexo seguro, prevención de ISTs, embarazo adolescente, autocuidado y la importancia de un estilo de vida saludable y debate sobre la violencia. Conclusión: El evento brindó a los estudiantes el contacto con la realidad social de las participantes, posibilitando la reflexión sobre la humanización de la salud de las mujeres y la comprensión de prácticas basadas en el diálogo, la recepción y la construcción colectiva.
\end{abstract}

Palabras clave: Salud de la mujer; Prevención de enfermidades; Educación en salud.

\title{
1. Introdução
}

A atenção primária à saúde da mulher é de fundamental importância devido à abrangência e peculiaridades dos cuidados nos diferentes momentos de vida, como por exemplo a gravidez, o puerpério, no climatério e frente a doenças específicas, que podem prejudicar gravemente a qualidade de vida da mulher e até mesmo levar ao óbito. Com isso, as políticas de saúde incluem ações educativas, preventivas, de diagnóstico, tratamento e recuperação, englobando a assistência à mulher em clínica ginecológica, no pré-natal, parto e puerpério, no climatério, em planejamento familiar, infecções sexualmente transmissíveis (IST), câncer de colo de útero e de mama, além de outras necessidades identificadas a partir do perfil populacional.

Nessa perspectiva, a Secretaria de Estado de Políticas para as Mulheres e a Área Técnica de Saúde da Mulher do Ministério da Saúde incentivam ações de promoção à saúde da mulher de forma integral, individual e coletiva, em todos os âmbitos da sociedade. A promoção da saúde da mulher só se concretiza na medida em que a mulher seja protagonista das ações que a beneficiam.

Observam-se diversas lacunas no que diz respeito à saúde integral feminina. Podemos citar, por exemplo, alguns fatores levantados por Rocha et. al. (2017), que influem para a não adesão de acompanhamento gravídico-puerperal, sendo esses relacionados a desigualdades sociais, regionais e econômicas, bem como as dificuldades relacionadas à idade e à baixa escolaridade sugerindo que mulheres em vulnerabilidade social estão mais propensas a receberem menos informação e, assim, negligenciarem elementos importantes de sua saúde.

Em outro parâmetro, o de prevenção a Infecções Sexualmente Transmissíveis (IST's), também pode-se observar a presença dos mesmos fatores. Segundo boletins epidemiológicos do estado de Goiás (2018), para as IST’s mais comuns (Sífilis e HIV/Aids), houve um crescente aumento do número de infectados nos últimos dez anos analisados. A partir das notificações do Centro de Testagem e Aconselhamento-CTA de um município do sudoeste goiano, do ano de 2004 a 2014, é evidenciado maior vulnerabilidade de mulheres jovens, solteiras e de baixa escolaridade, em relação ao risco de transmissão e aquisição de IST 's. Portanto, pode-se inferir que as ações de conscientização estão sendo insuficientes e há a necessidade de maior abordagem do tema com a população sensível (Magalhães et. al., 2018).

Nesse contexto, merecem atenção os fatores que levam a baixa adesão às ações de prevenção e detecção precoce do câncer de colo de útero. Em relação ao exame para diagnóstico precoce de câncer de colo de útero, observa-se que os maiores impasses estão em torno do baixo conhecimento das mulheres sobre o procedimento ou ainda receio e constrangimento (Dantas et.al., 2018). Falar abertamente sobre a importância da prevenção de câncer de colo de útero, do câncer de mama e da 
realização dos procedimentos, é um movimento de quebra dessas barreiras, contribuindo para que haja maior adesão às políticas públicas voltadas a essa vertente.

Por essas e outras lacunas encontradas no que tange a integralidade da saúde da mulher, podemos apontar, como um dos possíveis caminhos, as atividades educativas como forma de reflexão para o público alvo. O enfoque deve estar em promover a saúde de forma holística e integral, considerando que a mulher deve ser informada e orientada sobre autocuidado, para que de forma consciente e autônoma, gerencie as soluções para seus cuidados de saúde. Para esse fim, podem ser utilizadas ações coletivas de educação em saúde, que dialogam com a população feminina e toda a sociedade.

Nesse contexto de ações intersetoriais, a Comissão da Mulher Advogada, que foi criada em 2013 pela Ordem dos Advogados do Brasil (OAB) e está presente em suas seções e subseções, apresenta como objetivo promover a valorização da mulher advogada e contribuir ainda para a promoção da igualdade de gênero na sociedade. Dessa forma, é uma comissão que realiza diversos trabalhos e projetos em prol da mulher, pela sua valorização necessária na sociedade como um todo.

Em parceria com as instituições públicas e privadas, e de educação e ensino superior públicas, a Comissão da Mulher Advogada apresentou como uma necessidade a criação de um projeto buscando o empoderamento e valorização da mulher na cidade de Jataí-GO, voltado principalmente às mulheres que não possuem oportunidades de acesso à informação quanto aos seus direitos à saúde, família, proteção física, psicológica e intelectual, como forma de se resguardar dos vários tipos de violência que enfrentam no dia-a-dia.

As ações desenvolvidas nesse projeto, denominado Semana de Combate a Violência Contra a Mulher, constituem-se em eventos anuais, um deles sendo o OAB Mulher, que contam com palestras, feira de saúde e beleza, espaço jurídico e psicológico, espaço infantil, almoço e abertura para lazer no clube Thermas Jataí, proporcionando uma variedade de atividades para o público feminino, onde ocorrem várias parcerias fundamentais para a diversidade nas atividades realizadas, sendo uma delas a parceria com o Curso de Enfermagem da Universidade Federal de Jataí.

Para que a mulher se perceba como protagonista do seu cuidado em saúde, faz-se necessário criar oportunidades que permitam o contato e discussão sobre temas relevantes que influenciam positivamente em suas vidas. Estas oportunidades estão exemplificadas na presente iniciativa, como sendo atividades educativas, em cenários públicos frequentados por mulheres em diversas faixas etárias, de variadas classes sociais, com distintos níveis educacionais, diferentes profissões/ocupações, em diferentes condições de saúde, mas que devem estar permanentemente sendo informadas a respeito das ações de promoção de saúde oferecidas pelo Sistema Único de Saúde.

A motivação maior para o desenvolvimento do projeto foi acreditar, e levar futuros profissionais da Enfermagem a acreditarem, que toda mulher é capaz de realizar o seu autocuidado desde que bem informada e orientada para exercer sua autonomia. Nesse contexto, acredita-se também que esta mulher se torna um agente motivador e multiplicador de informações e orientações na comunidade onde reside.

Assim, essa ação de extensão universitária teve como objetivo desenvolver práticas educativas relativas à: prevenção e controle do câncer do colo do útero e de mama, autocuidado durante a gestação, aleitamento materno, planejamento familiar, prática do sexo seguro, prevenção de ISTs, gravidez na adolescência, importância de manter um estilo de vida saudável e debate sobre violência contra a mulher.

Nessa perspectiva, o presente trabalho teve como objetivo relatar a experiência e mostrar a percepção de acadêmicos de enfermagem na realização de uma ação de educação em saúde, referente à saúde da mulher realizado em um município do sudeste goiano.

\section{Metodologia}

Consiste em um estudo descritivo, do tipo relato de experiência, realizado por acadêmicos de Enfermagem da 
Universidade Federal de Jataí, sobre o evento OAB Mulher, ocorrido em Jataí. A ação ocorreu no mês de março de 2019, contabilizando um total de 10 horas de atividades com as mulheres participantes, além das horas necessárias ao preparo das atividades e do material.

A proposta metodológica do projeto/atividade educativa consistiu na apresentação e diálogo sobre temas relevantes (aqueles propostos na justificativa) relativos à saúde da mulher, em cenários públicos frequentados por mulheres com estratégias de abordagem em grupos, apresentando informações e orientações a partir de material previamente elaborado, suscitando discussões e esclarecimento de dúvidas.

De acordo com Paulo Freire, o processo educativo ocorre e está centrado na mediação educador-educando. Ao educador cabe mostrar ao educando que ele traz consigo uma gama de conhecimentos oriundos de suas experiências e ao educador é incumbida a tarefa de auxiliar na organização desses conhecimentos, relacionando os saberes trazidos pelo educando com os saberes escolares.

Para tanto, as estratégias das atividades foram pautadas nessa metodologia, onde se partiu do conhecimento popular sobre o tema e, em conjunto com os interessados, desenvolveu-se o aprendizado modificador da realidade. Ao trabalhar a aprendizagem dessa maneira, o método inova ao promover a horizontalidade na relação educador-educando, a valorização da sua cultura e da sua oralidade.

Na visão de Paulo Freire a educação deve ser capaz de promover a autoconfiança e toda ação educativa deve ser um ato contínuo de recriação e de ressignificar os significados enquanto condição de possibilidade para uma educação conscientizadora e libertadora, dentro de uma perspectiva contínua de diálogo e reflexão sobre a ação com o objetivo de ampliar a visão de mundo e a participação ativa do indivíduo em todas as esferas da vida em sociedade (Freire, 2015).

\section{Resultados e Discussão}

A Semana de combate a violência contra a mulher surgiu a partir de outro evento, sobre o Dia Internacional da Mulher, realizado pelo projeto Colmeia, abordando o tema da violência doméstica e familiar contra as mulheres e também oferecendo às mulheres de grupos vulneráveis da cidade a oportunidade de acesso a serviços de saúde, bem-estar, beleza e atendimento jurídico e psicológico.

O Projeto Colmeia: empoderamento feminino é fruto da união de forças de várias entidades da sociedade civil organizada e de instituições presentes na cidade de Jataí-GO e foi idealizado pela Comissão da Mulher Advogada da Subseção Jataí da Ordem dos Advogados do Brasil (OAB). (Lima et al., 2017).

A III Semana de combate a violência contra a mulher foi desenvolvida em parceria com a Comissão da Mulher Advogada da OAB/Goiás, a OAB-Goiás, o Tribunal de Justiça do Estado de Goiás, a Universidade Estadual de Goiás, o Instituto Federal de Goiás - IFG, e o Projeto Colmeia, com o apoio do Serviço Social do Comércio (SESC), da Caixa de Assistência dos Advogados de Goiás - CASAG, do Centro Universitário UNA, do Instituto Mix de Profissões, CRI Jataí, da Universidade Federal de Goiás, da Fundação Raízen, da Nena Carvalho consultoria de imagem e estilo, da CRC Construtora, e do $41^{\circ}$ Bimtz.

O evento tornou-se tradição na cidade e contou com o apoio da Secretaria Municipal de Educação e Secretaria Municipal de Transporte, que forneceu às participantes translado gratuito nos principais bairros da cidade, sendo que as rotas foram divididas em cinco trajetos: trajeto 01- Cidade Jardim I e II, Jacutinga e Região; trajeto 02- Estrela Dalva, Francisco Antonio, Vila Sofia e Região; trajeto 03- Setor Geda, Vila Fátima, Vila Olavo, Vila Progresso, Vila Santa Maria e Região; trajeto 04- Bairros Campo neutro, Jardim da Liberdade, Frei Domingos, Nova Esperança, Colmeia PARK, Mauro Bento, JD. Paraíso e Região e trajeto 05- Cohacol V, Jardim Floresta, Fã, Vila Luzia, Hermosa, José Bento,Conjunto Rio Claro 1,2 e 3, Epaminondas, JD. Goiás e Região. 
No percurso para o local de realização do evento, as participantes foram orientadas sobre a programação e foi distribuído um lanche. Ao chegarem no clube Thermas Jatahy, foram recepcionadas com uma área dedicada aos estandes das instituições parceiras, com várias temáticas e diferentes abordagens. Destacando-se as tendas de atendimento jurídico e psicológico, que foram separadas das demais, para preservação da privacidade de quem procurasse apoio. Após passarem por essa etapa, que ficava localizada na entrada do clube, foi liberado o acesso à área interna para o almoço e para acesso às piscinas do clube.

No desenvolvimento do presente projeto, estiveram envolvidos dez alunos de graduação e dois docentes do Curso de Enfermagem da UFJ. A equipe de enfermagem realizou ações voltadas à promoção da saúde, abordando a importância do autocuidado e da prevenção. Também foram disponibilizados materiais impressos, com orientações objetivas em linguagem acessível. Durante o atendimento, foi realizada educação em saúde voltada à prevenção e controle do câncer do colo do útero e de mama, autocuidado durante a gestação, aleitamento materno, planejamento familiar, a respeito da prática do sexo seguro, da prevenção de ISTs, gravidez na adolescência e importância de manter um estilo de vida saudável, também foi realizado o procedimento de aferição da pressão arterial.

Seguindo as atividades, no mesmo espaço, ocorreu uma ação de educação em saúde, a fim de promover o autocuidado da mulher com relação ao câncer de mama e colo do útero. Esta aconteceu por meio de uma conversa informal, clara e objetiva, em que se concernem as seguintes particularidades: importância do autoexame das mamas, salientando o principal objetivo da realização deste, incentivando para que as mulheres conheçam suas mamas, facilitando a percepção de qualquer alteração, que, porventura, possa aparecer.

Foram realizadas também orientações quanto a necessidade de atentar-se para pequenos nódulos nas mamas e na região das axilas, saída de secreções mamilares, mudança de cor da pele ao redor da mama e no tamanho ou no formato da mesma, dor nas mamas ou na região das axilas, a pele da mama apresentando-se com aspecto de casca de laranja, avermelhada, com prurido e com alterações na estrutura do mamilo. Ainda, foi informado que tais sinais e sintomas são sinais de alerta do câncer de mama.

Foi orientado que na ocorrência de alguma das alterações mamárias citadas acima, a mulher procure de imediato o serviço de saúde. Uma vez que o enfermeiro deve usufruir da ferramenta de trabalho da orientação para construir um atendimento mais humanizado. Logo, orientar significa deixar entendido conforme evolui a doença, as especificidades das etapas e como agir com cada uma delas, respeitando a realidade socioeconômica e a rotina de cada família (Santana; Almeida; Savoldi, 2019).

No término do esclarecimento sobre o câncer de mama, proporcionou-se uma dinâmica de palpação em mamas de borracha para a identificação de nódulos, com a perspectiva de oferecer o conhecimento de como estas se apresentam nos casos de neoplasias, evitando o surgimento de dúvida nos casos de identificarem o aparecimento desta alteração.

Além das orientações relacionadas ao câncer de mama, foi frisado sobre a importância do exame citopatológico e da sua realização periódica. Enfatizou-se que na realização deste o enfermeiro pode detectar alterações cervicais, que possam indicar uma neoplasia ou infecção sexualmente transmissível (IST), e que com o diagnóstico precoce destas patologias as chances de cura são bastante favoráveis.

As participantes também foram orientadas sobre as práticas adequadas para terem uma qualidade de vida melhor, informado a elas hábitos importantes de como ter um estilo de vida mais saudável no seu cotidiano. Ressaltamos a importância de ter hábitos saudáveis no dia a dia para evitar doenças crônicas como Diabete Mellitus e Hipertensão Arterial.

Sendo que os principais fatores de risco para a HAS incluem: hereditariedade, idade, raça, obesidade, estresse, vida sedentária, álcool, sexo, anticoncepcionais e alta ingestão de sódio. Outros fatores, tanto sociais quanto físicos, também são destacados, não por serem causadores da HAS, mas por estarem frequentemente associados a ela (baixo nível educacional, 
colesterol elevado e diabetes mellitus. (Virgínia et al., 2012).

$\mathrm{O}$ estilo de vida associado à prática de atividades físicas e uma boa alimentação pode levar o indivíduo a prolongar seus dias de vida, assim como evitar o desenvolvimento de doenças crônicas.

Assim como a maior longevidade da população, juntamente com as alterações no estilo de vida, sobretudo o sedentarismo e as mudanças no padrão de alimentação, contribuem para o aumento do perfil de risco para doenças crônicas, como o diabetes mellitus (DM). (Correia et al., 2016).

O primeiro contato com as participantes foi iniciado por meio de uma conversa informal, em que realizamos perguntas sobre os hábitos cotidianos em relação aos cuidados com a saúde. Perguntamos se tinha diabetes ou hipertensão ou familiares portadores, se tomava algum medicamento, se praticava atividades físicas, se consumiam álcool ou tabaco e com que frequência, e de como era a alimentação no dia a dia.

Através das respostas obtidas passamos as orientações relacionadas a cada pergunta. Sendo orientando sobre a importância de ter uma rotina para a prática de atividades físicas e aproveitar as oportunidades para realizá-las, também sobre o hábito de consumir alimentos saudáveis levando em consideração a realidade socioeconômica de cada indivíduo. E por meio dessa comunicação eram sanadas as dúvidas e curiosidades sobre os assuntos abordados durante a conversa.

Em seguida, foi aferida a pressão arterial, momento que aproveitamos para enfatizar as orientações passadas, falar da importância de colocá-las em prática no dia a dia e levar essas informações para seus familiares e amigos. Ao final do atendimento era entregue um panfleto com orientações sobre os temas abordados para que ficassem atentos aos sintomas e sempre procurar os atendimentos nas unidades básicas próximas de suas residências e também lembrando da importância de seguir o tratamento contínuo após ser diagnosticado com uma doença crônica.

Durante os atendimentos, percebeu-se que os assuntos relacionados à sexualidade ainda são tratados como tabu, pois ao observarem o material apresentado, a maioria das mulheres se mostrou acanhada. Na maioria dos casos, quando questionadas, se desviaram das perguntas. Algumas mulheres analisavam, à distância, demonstrando interesse pelo conteúdo exposto, porém, não se aproximavam, em alguns casos, os alunos se deslocavam para sanar algumas dúvidas. Aquelas que se abriam ao diálogo, demonstravam um conhecimento superficial e defasado em relação aos temas abordados.

Ao final de cada atendimento, foi entregue aos participantes preservativos de ambos os sexos, sendo demonstrado em peças anatômicas (levadas pelos próprios alunos), a forma correta de utilizá-los, ressaltando o desconhecimento relacionado ao preservativo feminino, em decorrência, obteve maior destaque na demonstração prática. Levando em consideração a experiência vivida, pode-se perceber, que eventos como esse, devem se tornar cada vez mais comuns, para que a informação chegue a todos os públicos, promovendo cada vez mais saúde de qualidade.

Pensando nisso, procurou-se, em todos os momentos da ação, criar um vínculo com a mulher e deixá-la o mais à vontade possível, favorecendo o desenvolvimento da atividade e a conscientização da mesma para a realização do exame citopatológico anualmente e o autoexame das mamas mensalmente. No final de cada atendimento, foi entregue pelos acadêmicos de enfermagem um cartão educativo, o qual ressalta a importância dos temas abordados no decorrer do evento. 
Figura 1 - Equipe de discentes, docentes e apoio da UFJ no projeto “Colmeia”, Jataí-GO, Brasil, 2019.

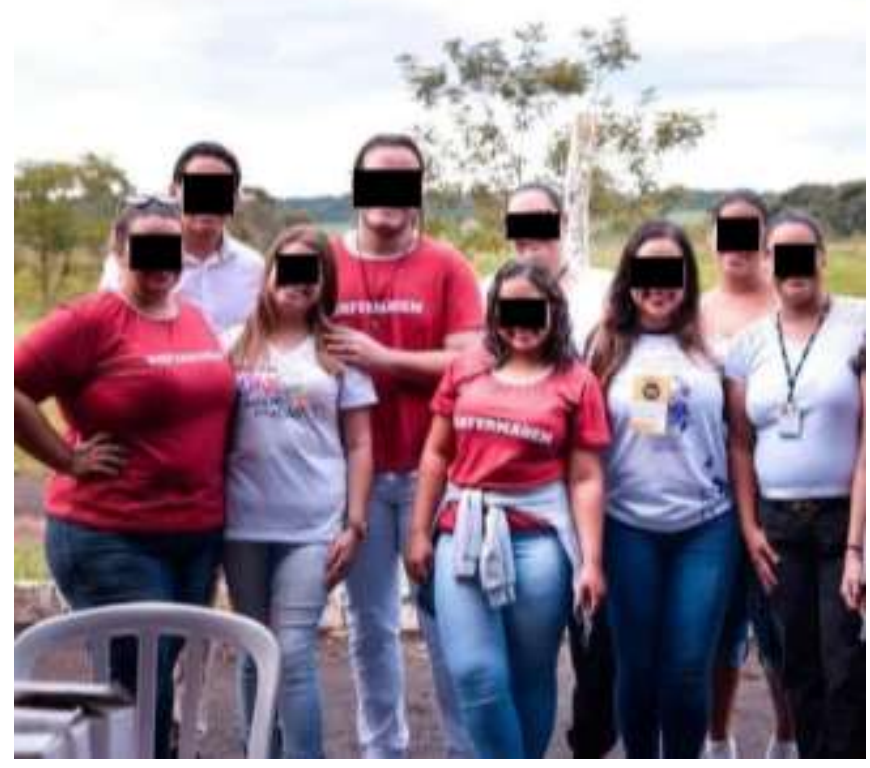

Fonte: Site do Projeto "Colmeia".

\section{Considerações Finais}

As ações realizadas tiveram como foco a educação em saúde, por meio de práticas que estimulam o exercício do autocuidado pelas mulheres. Logo, esta intervenção foi uma estratégia amplamente discutida e construída de forma coletiva, entre os acadêmicos de enfermagem, as professoras supervisoras, a secretaria municipal de saúde e a Comissão da Mulher Advogada.

A realização destas atividades possibilitou a troca de experiências entre os profissionais, acadêmicos e as mulheres participantes. A efetivação da ação possibilitou retomar a relevância do acolhimento, vínculo e, principalmente, das ações para a promoção da saúde da mulher e a prevenção de doenças, além de influenciar na formação do profissional enfermeiro, desenvolvendo nos discentes o senso de cidadania e responsabilidade social.

A ação em saúde desenvolvida com as mulheres foi uma oportunidade ímpar, possibilitando a inserção dos acadêmicos de enfermagem à realidade social das mulheres, colaborando para a reflexão no que se refere à atenção humanizada na saúde da mulher. Assim, se reconhece a necessidade de envolvimento dos acadêmicos em ações de extensão, a fim de incentivar a prática da promoção da saúde e prevenção das doenças nos diferentes ciclos de vida das pessoas, possibilitando que se tornem profissionais ativos no processo de educação em saúde.

\section{Referências}

Alves, V. S. Um modelo de educação em saúde para o programa saúde da família: pela integralidade da atenção e reorientação do modelo assistencial. Interface Comum, Saúde Educ., 9(16), 39-52.

Brasil. Ministério da Saúde. Secretaria de Atenção à Saúde, Departamento de Ações Programáticas Estratégicas. (2011). Política nacional de atenção integral à saúde da mulher: princípios e diretrizes.

Brasil. Ministério da Saúde. Secretaria de Atenção à Saúde, Departamento de Ações Programáticas Estratégicas. (2006). Estudo da mortalidade de mulheres de 10 a 49 anos, com ênfase na mortalidade materna: relatório final.

Brasil. Ministério da Saúde. Secretaria de Atenção à Saúde, Departamento de Atenção Básica. (2013). Saúde sexual e saúde reprodutiva.

Brasil. Ministério da Saúde. Secretaria de Atenção à Saúde, Departamento de Atenção Básica. (2012). Atenção ao pré-natal de baixo risco. 
Research, Society and Development, v. 10, n. 11, e276101119428, 2021

(CC BY 4.0) | ISSN 2525-3409 | DOI: http://dx.doi.org/10.33448/rsd-v10i11.19428

Brasil. Ministério da Saúde. Secretaria de Atenção à Saúde, Departamento de Atenção Básica. (2013) Controle dos cânceres do colo do útero e da mama. (2a ed.).

Brasil. Ministério da Saúde. Secretaria de Atenção à Saúde, Departamento de Ações Programáticas Estratégicas. (2008). Manual de atenção à mulher no climatério/menopausa.

Brasil. Ministério da Saúde. Secretaria de Vigilância em Saúde, Departamento de DST, Aids e Hepatites Virais. (2015). Protocolo clínico e diretrizes terapêuticas para atenção integral às pessoas com infecções sexualmente transmissíveis.

Brasil. Presidência da República. Secretaria Especial de Políticas para as Mulheres. (2013). Plano Nacional de Políticas para as Mulheres.

Carvalho, M. V., Siqueira, L. B., Sousa, A. L. L., \& Jardim, P. C. B. V. A influência da hipertensão arterial na qualidade de vida. Arquivos Brasileiros de Cardiologia, 100(2), 164-174.

Cervera, D. P., Parreira, B. D., Goulart, B. F. Educação em saúde: percepção dos enfermeiros da atenção básica em Uberaba (MG). Ciências Saúde Coletiva, $32(25), 56-65$.

Czeresnia, D. O conceito de saúde e a diferença entre prevenção e promoção. Cadernos de Saúde Pública In: Czeresnia D, Freitas CM (org.). Promoção da Saúde: conceitos, reflexões, tendências.: Ed. Fiocruz, 2018.

Corrêa, K., Gouvêa, G. R., Silva, M. A. V. da, Possobon, R. F., Barbosa, L. F. L. N., Pereira, A. C., Miranda, L. G., \& Cortellazzi, K. L. Qualidade de vida e características dos pacientes diabéticos. Ciência \& Saúde Coletiva, 22, 921-930.

Dantas, P. V. J., Leite, K. N. S., César, E. S. R., Silva, S. C. R., Souza, T. A. de, \& Nascimento, B. B. do. Conhecimento das mulheres e fatores da não adesão acerca do exame papanicolau. 2018. Rev. Enferm. UFPE on line. 12, 684-91, 2018.

Freire, P. (2015). Pedagogia da autonomia: saberes necessários à prática educativa. (51a ed.): Paz e Terra.

Lima, V. K.S., Hollanda, G. S. E., Oliveira, B.M., Oliveira, I. G., Santos, L. V. F., Carvalho, C. M. L. Educação em saúde para gestantes: a busca pelo empoderamento materno no ciclo gravídico-puerperal. Revista de Pesquisa: Cuidado é Fundamental Online, 18(22), 144-155 2020.

Magalhães, A. P., Souza, G. M. de, Borges, C. J., Moraes, L. C., Souza, M. R. de, Santos, O. P. dos, \& Barros, P. S. Perfil dos usuários do centro de testagem e aconselhamento de um município de sudoeste do estado de Goiás, 2004-2014. Itinerarius Reflectionis, 14(4), 01-22.

Oliveira, E., Andrade, I. N., Ribeiro, R. S. Educação em saúde: uma estratégia da enfermagem para mudanças de comportamento. Universidade Católica de Goiás. 3(10), 114-123.

Rocha, I. M. da S., Barbosa, V. S. de S., \& Lima, A. L. da Silva. Fatores que influenciam a não adesão ao programa de pré-natal. Revista Recien-Revista Científica de Enfermagem, 7(21), 21-29.

Santana, R. F., Almeida, K. S., \& Savoldi, N. A. M. Indicativos de aplicabilidade das orientações de enfermagem no cotidiano de cuidadores de portadores de Alzheimer. Rev Esc Enferm. USP, (43a ed.), 459-464. 